\title{
PETROLE, ENERGIES RENOUVELABLES ET LA DISPUTE AU NIVEAU FEDERAL AU BRESIL
}

\author{
PETRÓLEO, ENERGIAS RENOVÁVEIS E DISPUTA À NÍVEL FEDERAL NO BRASIL
}

\author{
Angela Moulin S. Penalva Santos ${ }^{1}$
}

\section{Resumo}

O Brasil está organizado como uma federação trina desde que, em 1988, os municípios tornaram-se entes federativos. O fortalecimento institucional do Município incluiu a responsabilidade pela execução das políticas sociais, que eles são obrigados a co-financiar. Ao longo do tempo, a maior gama de responsabilidades administrativas e financeiras tem indicado os limites da descentralização das políticas públicas já que a maior parte dos municípios não tem condições de atender às expectativas criadas com o seu papel na estrutura federativa. Trata-se de um problema derivado do federalismo simétrico no Brasil, que trata igualmente todos os municípios, sem distingui-los por porte demográfico ou função na rede urbana.

Esse é o contexto em que se estabelece a disputa federativa pela crescente receita que as empresas pagam ao poder público pela extração de petróleo. Tal receita deixa de ser percebida como compensação pelos impactos ambientais e sociais causados pela atividade extrativa e torna-se mais uma transferência intergovernamental aos entes federativos. Tal situação tende a torna-los todos mais dependentes desta fonte de receita e, assim, mais sensíveis aos interesses da economia do petróleo, o que diminui o ímpeto governamental na defesa da política de substituição de energia fóssil por renováveis.

Palavras chave: petróleo, energias renováveis, disputa, federação.

\begin{abstract}
Brazil is organized as a federation in three levels including the federal union, states and municipalities since 1988 when municipalities have become federal entities. The institutional strengthening of the municipality included the responsibility for the implementation of social policies which they are required to co-finance. Over time, the larger range of administrative and financial responsibilities has indicated the limits of decentralization of public policies since most municipalities cannot afford to meet the expectations created by its role in the federal structure. It is a problem of the symmetric federalism in Brazil, which treats all municipalities without distinguishing them by population size or function in the urban network. This is the context of the dispute by the increasing federal revenues that companies pay to the government from oil. This income is no longer seen as a compensation for environmental and social impacts caused by extractive activity and becomes an intergovernmental transfer to states and municipalities. This situation tends to make them all more dependent on this source of revenue and thus more vulnerable to the interests of the oil economy, which reduces the government interests to defend the replacement policy of fossil energy by renewable.
\end{abstract}

Keywords: oil, renewable energy, dispute federation.

\footnotetext{
${ }^{1}$ Professeur Associé de I'Université de l'Etat du Rio de Janeiro - UERJ. E-mail: angelapenalva@terra.com.br
} 


\section{Résumé}

Depuis 1998 date à laquelle les Municipalités ont été consacrées en tant qu'entités fédérées, le Brésil est organisé comme une fédération à trois niveaux. Le renforcement institutionnel de la Municipalité lui confère également l'obligation d'exécuter des politiques sociales et, par conséquent, de les cofinancer. Au fil du temps, une plus grande part de responsabilités administratives et financières ont prouvé les limites de la décentralisation des politiques publiques. La plupart des Municipalités n'ont pas les moyens de répondre aux expectatives issues de leur nouveau statut au sein de la fédération. II s'agit d'un problème qui dérive du fédéralisme symétrique brésilien, dans lequel les Municipalités sont traitées de façon isonomique, en dépit de leur envergure démographique ou de leur fonction dans le réseau urbain.

Dans ce contexte, les conflits fédératifs se forment, en vue de l'obtention des ressources croissantes que les entreprises paient au pouvoir public en fonction de la prospection du pétrole. Cette recette n'est plus distinguée considérée comme une compensation des impacts environnementaux et sociaux provoqués par les activités de prospection; elle devient un transfert gouvernemental entre les unités de la fédération. Ce cadre augmente la dépendance de ces dernières à ces sources financières et les rend ainsi plus vulnérables aux intérêts de l'économie du pétrole, réduisant l'impact gouvernemental des politiques de substitution de l'énergie fossile par les énergies renouvelables.

Mots-clés: pétrole, les énergies renouvelables, litige, fédération.

\section{INTRODUCTION}

Cet article s'intègre au sein d'un vaste projet dont l'objectif est d'analyser la politique urbaine dans le contexte de la fédération brésilienne. Le fédéralisme fiscal sera ici la perspective principale. Ce dernier a été plutôt ébranlé par les recettes de pétrole, qui ont mis les entités fédérées en compétition pour leurs rendements, en provoquant le changement des normes de régulation ainsi qu'un engagement plus fort des politiques publiques brésiliennes avec la matrice énergétique fossile. Par conséquent, les énergies renouvelables ont été reléguées à une position moins importante.

Le Brésil est organisé comme une fédération tripartite, constituée de de l'Union, de 27 Étatsmembres (I'un d'entre eux étant le District Fédéral) et de 5.570 Municipalités, tous considérés comme des "entités fédératives», pourvues d'une autonomie politique, législative, administrative et financière. D'un point de vue juridique, il n'existe pas de hiérarchie entre ces entités. Toutefois, dans une perspective qui prend en compte l'autonomie financière, un fort déséquilibre est observable. Le fait que I'Union répond d'approximativement $60 \%$ des dépenses publiques, tandis que les Etatsmembres et les Municipalités répondent, respectivement, de $25 \%$ et $15 \%$, est un exemple de cette inégalité (Santos, 2008).

La Constitution de 1988 a fortifié les Municipalités au niveau institutionnel en les qualifiant expressément comme formant une entité fédérative. La Constitution a également transféré à cette échelle de gouvernement la responsabilité de l'exécution des politiques sociales, les obligeant à co- 
financier ces dernières. Au fil du temps, une plus grande quantité de responsabilités administratives et financières a révélé les limites de la décentralisation des politiques publiques, étant donné que la plupart des Municipalités n'ont plus les moyens de répondre aux expectatives créées suite à leur renforcement institutionnel dans la structure fédérative.

Il s'agit d'un problème provenant du fédéralisme symétrique brésilien, lequel consacre un traitement isonomique à toutes les Municipalités, sans les distinguer de leur taille démographique ou par la fonction qu'elles réalisent dans le tissu urbain. 70\% des Municipalités brésiliennes comptent avec une population inférieure à 20 mille habitants et ce groupe représente approximativement 20\% du PIB national. À savoir, la plupart des Municipalités est constituée d'une population à faibles revenus, n'ayant pas les moyens d'augmenter leur budget par l'augmentation de leurs propres charges fiscales. Donc elles dépendent beaucoup des transferts de ressources provenant d'autres échelles du » gouvernement.

C'est dans ce contexte que la dispute fédérative s'établit pour la croissante recette des royalties et des "participations spéciales", que les entreprises paient aux pouvoirs publics pour que l'exploitation du pétrole ait lieu. De surcroît, ces valeurs sont calculées à partir des prix internationaux, qui se sont maintenus à des niveaux élevés durant les 15 dernières années. De ce fait, les recettes des royalties et des " participations spéciales » - ne sont pas considérées comme une compensation pour les impacts environnementaux et sociaux provoqués par l'activité d'extraction et les recettes deviennent simplement un transfert intergouvernemental que toutes les entités fédérées méritent. Cette situation a tendance à rendre les entités fédérées plus dépendantes de cette source de recettes et, en conséquence, plus sensibles aux intérêts de l'économie du pétrole. Ce qui affaiblit la volonté du gouvernement de défendre la politique de substitution de l'énergie fossile par les énergies renouvelables.

Cet article est divisé en six sections, la première étant la présente introduction. La deuxième vise à situer l'importance des énergies renouvelables dans les politiques publiques. Dans un troisième temps, il conviendra d'examiner l'impact provoqué par la découverte dans la couche saline des fonds marins (zone " pre-salt ») le long de la côte. La quatrième section aura pour objet la contextualisation de l'impact du pre-salt dans les disputes fédératives. Par la suite, dans la cinquième section, le renforcement du gouvernement national sera associé au modèle du neo-devéloppement, qui donne la priorité aux droits sociaux par rapport aux droits diffus - comme la protection de l'environnement. Pour finir, des réflexions conclusives seront présentées.

\section{LES ÉNERGIES RENOUVELABLES NE SONT PAS LA PRIORITÉ AU BRÉSIL}


Quelle est la priorité des énergies renouvelables face à l'exploitation du pétrole ? Lors d'une interview pour le journal "O Globo » (édition du 13/10/2014), M. Tasso Azevedo a affirmé que le Brésil possède « le plus grand potentiel du monde pour générer de l'énergie solaire, éolienne et de biomasse. Ces dernières recevront $\mathrm{R} \$ 20$ milliards d'investissements pour les prochaines années. Le pétrole, à son tour, encaissera $\mathrm{R} \$ 700$ milliards pendant la même période $»$. II s'agit d'une évaluation critique de l'un des coordinateurs de l'Observatoire du Climat (réseau de 30 ONG qui surveille les changements climatiques), suite à son expérience au sein du gouvernement fédéral, de 2003 à 2009, lorsqu'il était responsable du Programme National des Forêts.

La différence de valeurs ne doit pas être interprétée comme la représentation de la différence de priorité des politiques énergétiques. L'exploitation du pétrole à des niveaux très profonds demande beaucoup plus d'investissements pour la recherche et le développement que les énergies renouvelables. Toutefois, la différence suggère un fort déséquilibre du poids politique des sources renouvelables.

Il convient donc de placer les choix énergétiques dans le contexte de choix adoptés par l'Etat. Quelle est la raison qui justifie cette concentration de financements dans le pétrole, une énergie fossile non-renouvelable?

Après un fort impact négatif provoqué par les deux crises mondiales du pétrole dans les années 70 (1973 et 1979) sur l'économie brésilienne, le gouvernement fédéral a décidé d'intensifier les efforts dédiés à l'exploitation du pétrole, de façon à diminuer la dépendance aux importations de cette source d'énergie. Quand les grands bassins de pétrole dans la mer territoriale ont été découverts, en direction de la côte de l'Etat de Rio de Janeiro, le pays n'a pas uniquement augmenté sa production interne, mais a également développé une importante capacité technologique d'exploitation offshore, ce qui a fait de l'entreprise brésilienne Petrobrás un important player international.

Sous le régime militaire (1964-1985), non seulement l'exploitation du pétrole s'intensifié, mais des recherches concernant les sources alternatives d'énergie ont été développées. L'arrêté n. 76.593 du 14/11/1975 a institué le projet du PROALCOOL, un programme dont l'objectif était de remplacer l'essence (gazoline) par l'alcool éthylique. L'objectif était de diminuer la dépendance du pays au pétrole importé, ainsi que de diminuer l'émission de gazes polluants.

En plus de réduire la consommation d'une source dérivée du pétrole, le programme de soutien à la production de l'éthanol -dénomination attribuée à l'alcool éthylique- a soutenu une activité agricole séculaire qui était alors en crise dans la région nord-est. A la fin des années 80, l'éthanol est devenu le carburant de la plupart des automobiles du Brésil. 
Cependant l'offre n'a pas accompagné la forte demande d'éthanol comme carburant, en provoquant le déstockage et menaçant la substitution par l'essence, dont le rendement était supérieure à celui de l'éthanol. La perte de compétitivité du prix de l'éthanol par rapport à celui l'essence, est le facteur principal qui explique la diminution de d'alcool. De plus, l'augmentation de la cotation du sucre a fait que les producteurs ont préféré orienter leur production vers des finalités agricoles et non pas énergétiques. Dans ces conditions, les recherches concernant l'augmentation de la production de l'éthanol n'ont pas présenté de régularité. Le Brésil perdrait, ainsi, son avantage initial de l'emploi de cette énergie renouvelable.

Le rôle protagoniste brésilien concernant l'emploi de cette biomasse comme source d'énergie dépendait de la manutention soit des prix élevés du pétrole, soit de l'une des subventions à la production de la canne à sucre. Mais le gouvernement n'est pas capable de contrôler la cotation du pétrole, une commodity négociée sur les marchés internationaux. Quand le prix du pétrole a chuté, le gouvernement fédéral a commencé à introduire un pourcentage d'éthanol dans l'essence (25\%), ayant ainsi l'ambition de soutenir la production des usines de canne à sucre. Les consommateurs ont recommencé à utiliser l'essence comme carburant, bien que les fabricants à l'industrie automobile aient continué leurs recherches en technologie, ce qui leur a permis de développer des voitures dont le moteur accepte les deux carburants: l'éthanol et l'essence (cette dernière avec un mélange d'éthanol).

Ces mesures n'ont pas été suffisantes pour maintenir en activité les usines d'alcool, qui ont été créés sous la fomentation du Programme PROALCOOL. Les anciennes régions de production qui n'ont pas accompagné le développement technologique et qui présentent une production moins représentative, régressent face à la compétition avec les usines plus modernes situées dans l'Etat de São Paulo. La durabilité de la production d'éthanol a été affectée plus négativement parce que, depuis la crise financière internationale de 2008, le gouvernement fédéral a décidé de subventionner l'essence. L'objectif étant d'atténuer les effets de cette crise et de mantenir des emplois de l'industrie automobile.

Si d'une part l'objectif du gouvernement a été atteint, d'autre part il a provoqué l'aggravation des problèmes environnementaux. L'augmentation de la flotte de voitures est le résultat de la conjugaison de facteurs comme l'augmentation des revenus du travail, une plus large disponibilité de crédit et l'allégement fiscal sur cette production. Cependant, cela a contribué à l'intensification de la crise urbaine, étant donné que les embouteillages ont été devenus plus chers, plus longs et plus pénibles. D'une perspective environnementale, le résultat n'a pas été plus grave parce que l'industrie automobile a commencé à employer des technologies moins polluantes par rapports aux années précédentes. 


\section{LE PRÉ-SEL ACCROÎT LA PRIORITÉ DU PÉTROLE COMME SOURCE D’ÉNERGIE}

Avant la crise, le gouvernement fédéral avait déjà fait marche arrière concernant sa politique d'énergies renouvelables, quand une réserve de pétrole assez conséquente a été découverte en 2007. II s'agit d'une aire de 800 kilomètres de longueur sur 200 kilomètres de large, à 300 kilomètres du littoral, depuis l’Etat du Espírito Santo jusqu'à l’Etat de Santa Catarina. «Pour aller de la superficie de l'eau jusqu'au sommet de la couche du pré-sel, au fond de la mer, il faut descendre à 5 mille mètres. Et pour arriver à la base de la formation saline, 2 mille mètres supplémentaires sont nécessaires. La quantité d'eau et de pierres de sel équivaut à une montagne de la dimension de l'Everest » (Dieguez, 2013, p. 217). La Petrobrás révèle qu'il s’agit de du plus grand gisement pétrolier découverte au cours de ces trois dernières décennies.

Selon Tosi (2013, pg. 87), « le volume de réserves de pré-sel suscite des polémiques. Le gouvernement affirme que le volume total des réserves varie entre 70 e 100 milliards de barils d'huile - équivalant entre pétrole et le gaz naturel (Petrobrás, 2011). Ce volume représente de 4 à 6 fois le volume actuel de réserves prouvées par la Petrobrás en 2012 ». Le début de l'exploitation de ces réserves signifierait la possibilité d'élever jusqu'à 40\% l'exploitation du pétrole par le pays (Piquet, 2011). Cela ferait du Brésil l'un des principaux producteurs de pétrole, en le plaçant dans une position qui lui permet d'entrer en concurrence avec les grands pays exportateurs actuels.

Il s'agit, toutefois, d'un défi technologique et financier de grandes proportions, étant donné qu'il concerne des zones situées des eaux profondes, englobant de nouvelles frontières technologiques et des solutions logistiques d'innovation par rapport aux activités liées à l'exploitation et à la production.

Piquet (2011) affirme que «dans les investissements dans les projets d'ingénierie, construction, montage de navires-sonde, plateformes, infrastructure logistique, ainsi que dans l'offre de services spécialisés, seront réalisés les plus larges bénéfices économiques et sociaux de la production nationale de pétrole et gaz; ceci présente des effets multiplicateurs au niveau de l'économie, de génération de revenus, d’emplois et de savoir-faire » (p. 83).

C'est de l'expérience norvégienne que provient l'exemple de la façon dont la découverte du pré-sel peut conduire la croissance de l'économie brésilienne. La Norvège a perfectionné son industrie 
dans les années 70, après la découverte du pétrole dans la Mer Nord. C'est la raison pour laquelle le gouvernement brésilien a introduit l'obligation d'un niveau minimal de résultat de la chaine de production. Le but est d'éviter que l'exploitation minérale soit uniquement une enclave dans l'économie brésilienne, selon les cas des pays comme le Venezuela et le Nigeria.

Les effets de l'enchainement provoqués par l'exploitation de pétrole dans la mer expliquent la valeur élevée des investissements qui sont réalisés dans l'économie brésilienne, comme l'explique Tasso Azevedo - cité ci-dessus. Corroborant cet impact sur les investissements publics, une étude du BNDES sur les perspectives d'investissements dans les secteurs industriels pour les années 2011 à 2014 a révélé que le secteur du pétrole et celui du gaz répondent par 61,5\% du total du pays (Puga \& Borça Jr, 2011).

C'est la Petrobrás, une société d'économie mixte contrôlé par l'Etat, qui commande tout le développement technologique et financier des investissements dans le secteur du pétrole et du gaz. L'entreprise, fondée en 1953 et dotée de pouvoirs de monopole pour exploiter le pétrole, a expérimenté une grande évolution technologique avec la découverte d’une importante province pétrolière sur la côte maritime brésilienne dans les années 70. En 1997, l’entreprise a perdu le monopole, quand la Loi n. 9.478/1997 est entrée en vigueur. Le nouveau cadre juridique a institué le régime des concessions des blocs aux entreprises qui voudraient exploiter du pétrole dans le pays, ce qui a attiré les grandes entreprises pétrolifères qui opèrent sur le marché international. Toutefois, l'expertise de la Petrobrás a été reconnue par ces entreprises, lesquelles ont préféré s'unir à l'entreprise brésilienne pour des finalités d'exploitation plutôt que d'entrer en concurrence avec cette dernière. Ce nouveau régime légal a provoqué l'augmentation de la production, en provoquant la fin de la dépendance de l'importation de l'huile. Les découvertes du pré-sel, toutefois, ont fait que le gouvernement brésilien change les conditions de régulation, avec l'objectif de prend en possession une parcelle plus grande des recettes de pétrole.

Quelle modification a été introduite dans la législation du pétrole ? Selon Serra \& Vilani (2012), le nouveau cadre de régulation est basé sur quatre objectifs principaux, lesquels se résument en trois lois fédérales, approuvées au cours de l'année 2010 (lois n. 12.351/2010, 12.304/2010 et 12.276/2010) : i) la création du régime de partage de production, à la place du régime de concession aux entreprises; ii) la création du Fond Social comme bénéficiaire de la recette gouvernementale du partage de production; iii) la création de l'entreprise Pétrole Pré-Sel S.A. (PPSA), la déléguée du gouvernement dans les consortiums créés pour le nouveau régime de partage de production ; et iv) l'apport financier à la Petrobrás, amplifiant la participation de l'Etat avec le droit de voter pour les décisions. 
Il convient de mettre en évidence que, dans ce scénario, la Petrobrás a assumée un rôle de protagoniste encore plus grand que celui qu'elle possédait avant que le monopole de production prenne fin. Nonobstant, les nouvelles normes ont créé des obligations qui ont laissé la Petrobrás plus vulnérable sur le marché de compétition où l'entreprise opère. Cela s'explique parce qu'elle est devenue l'opératrice de tous les blocs qui sont offerts dans ce nouveau régime de partage, en lui garantissant une participation minimale de 30\%. Serra \& Vilani (2012) expliquent que, dans les cas d'appel d'offre d'un bloc d'exploitation, l'entreprise gagnante sera obligée de constituer un consortium avec la PPSA et la Petrobrás (cette dernière avec une participation non-inférieure à 30\%). Dans ces conditions, le rythme de la prospection du pétrole irait déterminer les compromis financiers de l'entreprise. Celui-ci est un des arguments que les détracteurs du changement du cadre de régulation de l'exploitation du pétrole posent pour expliquer pourquoi les appels d'offre des nouvelles aires d'exploitation ont été suspendus en 2007 et reprises uniquement en 2014, bien que la nouvelle loi soit en vigueur depuis 2010.

Le président de l'Agence Nationale de Pétrole (ANP), Haroldo Lima, pendant la période de découverte de la couche du pré-sel, avait admis qu'il était problématique d'insérer dans la législation une prévision expresse que la Petrobrás participerait à toutes les opérations, même dans les blocs où elle serait uniquement une partenaire d'une autre entreprise de pétrole (Dieguez, 2014). L'expansion des recettes de pétrole, d'après lui, résulte principalement de l'augmentation des prix du pétrole: « quand la loi du pétrole a été réglementée, le prix du baril valait environ 17 dollars. Aujourd'hui ${ }^{2}$, il coute 110 , la rentabilité de tous les puits s'est multipliée (p. 234). Pour cette raison, l'ex-président de I'ANP jugeait que, plutôt que de changer les normes de régulation, l'augmentation du pourcentage des participations sociales serait suffisante.

Mais le gouvernement a vu dans la découverte du pré-sel l'opportunité d'augmenter augmenter son contrôle sur une richesse qui pourrait financer le développement du pays. Comme cette découverte était le fruit des recherches de la Petrobrás, conclure les contrats de concessions avec d'autres entreprises de pétrole pour l'exploitation dans les aires dont les réserves étaient déjà confirmées, équivalait à " donner un ticket gagnant aux concurrents», selon les mots de Guilherme Estrella, qui était alors le directeur d'exploitation et de production de la Petrobrás (Dieguez, 2014, pg.220)

Le gouvernement a décidé de changer la base de régulation et de rendre la Petrobrás l'actrice principale de l'exploitation du pétrole. L'entreprise avait déjà donné des exemples de son expertise en

2 Interview donné à Consuelo Dieguez, publié dans la Revue Piauí en septembre 2012. Celle-ci et d'autres investigations de l'auteur ont été réunies dans l'ouvrage Bilhões e Lágrimas e A economia brasileira e seus atores, en 2014. 
recherche et développement. Reste encore à confirmer sa capacité de financer un grand nombre d'opérations simultanées, en cas d'effective réalisation des investissements prévus.

\section{LA DISPUTE POUR LE PÉTROLE, AU NIVEAU DE LA FÉdERATION}

« Entre 1998 et 2012, l'allocation des royalties de pétrole et de participations spéciales à dix États-membre et 823 Municipalités a dépassé le seuil d'un milliard de reais (R\$) pour 90 milliards de reais (Dieguez, 2014, pg. 265). II s'agit des de valeurs extraordinaires, qui équivalent respectivement à 400 mille dollars et 36 milliards de dollars. L'impact de l'économie du pétrole sur les finances publiques a eu tendance à augmenter en raison de l'expansion de l'extraction et du prix du pétrole. Quand le pré-sel sera en opération, ces valeurs doivent se multiplier.

La perspective d'une recette croissante, provenant de l'exploitation du pétrole, a provoqué des questions également par rapport à la distribution des royalties aux entités de la fédération. Dans le régime légal institué en 1997, la recette du pétrole était partagée de manière à ce que l'Union, les Etats-membres et Municipalités qui le produisaient en bénéficient. Cela signifie une forte concentration de recettes dans l'Etat du Rio de Janeiro et dans certaines des Municipalités, comme le montre le tableau ci-après.

Tableau 1: Distribution annuelle des recettes du pétrole (royalties et participations spéciales), 2010. Evidences de concentration spatiale.

\begin{tabular}{|c|c|c|}
\hline Bénéficiaire & US\$ Milliards & $\%$ \\
\hline Brésil & 12,27 & 100 \\
\hline Union & 4,91 & 40 \\
\hline $\begin{array}{c}\text { États-membres } \\
\text { (26 États-membres + District Fédéral) }\end{array}$ & 4,41 & 36 \\
\hline Etat de Rio de Janeiro & 3,64 & 30 \\
\hline $\begin{array}{c}\text { Municipalités brésiliennes } \\
\text { (5.565 au total) }\end{array}$ & 2,95 & 24 \\
\hline $\begin{array}{c}\text { Municipalités dans l'Etat du Rio de Janeiro } \\
\text { (92 Municipalités au total) }\end{array}$ & 1,90 & 15 \\
\hline
\end{tabular}

Source : élaboré par Serra \& Vilani (2012) avec les données de l'Agence Nationale de Pétrole.

La législation de partage des recettes de pétrole est entrée en vigueur depuis l'année fiscale 1998, mais elle a commencé à engendrer un volume expressif de recettes dans la mesure où les concessions réalisées ont provoqué l'augmentation de la prospection de pétrole, dans un contexte d'élévation des prix internationaux. La preuve peut en être observée dans les tableaux suivants.

Tableau 2 : Evolution de la recette de pétrole par rapport au PIB brésilien au cours d'années choisies. En \%. 


\begin{tabular}{|c|c|c|c|}
\hline $\begin{array}{c}\text { Année/Munici } \\
\text { palité }\end{array}$ & $\begin{array}{c}\text { Municipalités de } \\
\text { L'Etat de Rio de } \\
\text { Janeiro (A) }\end{array}$ & $\begin{array}{c}\text { Municipalit } \\
\text { és } \\
\text { Brésiliennes } \\
(B)\end{array}$ & $(A) /(B)=\%$ \\
\hline 2002 & 0,0039 & 0,0125 & 30,9 \\
\hline 2004 & 0,04 & 0,0724 & 55,2 \\
\hline 2008 & 0,084 & 0,1398 & 60,1 \\
\hline 2010 & 0,049 & 0,0926 & 52,9 \\
\hline 2012 & 0,0849 & 0,1441 & 58,9 \\
\hline
\end{tabular}

Source : Elaboration propre, partant des données du Secrétariat du Trésor National.

Au long de cette période mentionnée ci-dessus (après 1998), la participation de l'Etat du Rio de Janeiro aux recettes du pétrole (royalties et participations spéciales) s'est maintenue à un degré supérieure à 50\% par rapport aux valeurs qui ont augmenté de façon expressive proportionnellement au changement du PIB. II faut mettre en évidence, de plus, que la valeur do PIB a augmenté au cours de cette même période, ce qui suggère une augmentation encore plus grande de la collecte des recettes des entreprises de pétrole. Le tableau suivant présente le poids de ces valeurs dans les recettes budgétaires des Municipalités de l'Etat de Rio de Janeiro, ainsi que des autres États-membres.

Tableau 3 : Evolution de la collecte de recette de pétrole comme pourcentage des recettes des Municipalités pour les années choisies. En \%

\begin{tabular}{|c|c|c|}
\hline Année/Municipalité & $\begin{array}{c}\text { Municipalités de l'Etat du } \\
\text { Rio de Janeiro }\end{array}$ & Municipalités brésiliennes \\
\hline 2002 & 0,46 & 0,17 \\
\hline 2004 & 4,90 & 1,10 \\
\hline 2008 & 10,72 & 1,68 \\
\hline 2010 & 6,12 & 1,13 \\
\hline 2012 & 9,50 & 1,66 \\
\hline
\end{tabular}

Source : Elaboration propre partant des données du Secrétariat du Trésor National

L'impact des recettes du pétrole sur les rendements des Municipalités a fortement augmenté après la Loi n. 9.478/97. Au moment où elle entrée en vigueur, durant l'année fiscale de 1998, le pourcentage de recettes des royalties s'est élevé de $5 \%$ à 10\%. Cependant, l'impact principal a été effet de l'obligation de payement des dites «participations spéciales ». Ces dernières se rapportent aux impositions fiscales qui dépassent la moyenne du pétrole prospecté, situation qui s'est produite avec les blocs les plus fructueux, situés sur la côte maritime de l'Etat du Rio de Janeiro. La loi stipulait que la plus grande partie des prélèvements de ces recettes reviendrait aux Municipalités frontalières, 
à savoir les Municipalités situées « face » aux puits de pétrole, et, en second lieu, aux Municipalités qui abritent les installations pour le transport de l'huile.

Cette législation a fortement profité à quelques Municipalités de l'Etat de Rio de Janeiro, en leur intitulant même le surnom "les émirats fluminenses ». Cela s'attribue plutôt à l'impact de l'économie du pétrole sur les finances municipales - et de l'Etat du Rio de Janeiro - qu'à la chaîne de production provenant de l'extraction de cette richesse minérale. De cette façon, lorsque les découvertes de la couche du pré-sel ont été annoncées, la perspective d'augmentation des recettes a conduit l'Union à changer la législation pour que les richesses obtenues soient distribuées d'une façon plus équitable. Elle a aussi provoqué une dispute fédérative, empêchant la concentration de cette richesse au sein de quelques Municipalités - et également dans quelques Etats-membre. La conséquence en a été le changement de la législation qui fixait la distribution inter-fédérative des recettes de pétrole.

Contrairement aux trois autres lois déjà mentionnées - qui fixent le nouveau régime légal concernant l'extraction du pétrole, approuvées en 2010 - le Congrès National a trouvé plus de difficultés pour décider la manière de distribuer les revenus entre les entités fédérées. II a approuvé une nouvelle loi (n. 12.734/2012) qui redistribue les recettes entre tous les Etats-membres et Municipalités, indépendamment de la condition de producteurs ou non producteurs de pétrole. De plus, la loi a prévu l'application de ce régime même pour les contrats de concession signés auparavant, quand l'ancien régime de distribution de recettes était encore en vigueur, profitant davantage aux Etas-membres et aux Municipalités considérés comme « producteurs».

La Présidente de la République a mis son veto sur l'article de la Loi n. 12.734/2012 traitant de la redistribution des recettes du pétrole, et considéré comme étant inconstitutionnel, parce qu'il englobait les contrats antérieurs à la période de validité de la loi. Toutefois, les législateurs ont annulé le veto présidentiel lors d'une votation au parlement. C'est alors que les Etats-membre de Rio de Janeiro et d'Espírito Santo - autre important producteur de pétrole - ont intenté une action de contrôle de constitutionnalité en justice, devant le Suprême Tribunal Fédéral -la cour constitutionnelle brésilienne-, par l'intermédiaire de l'Action Directe de Inconstitutionnalité-ADI n. 4.916).

Une décision préliminaire dans la procédure référée a maintenu en vigueur la loi précédente pour les contrats passés avant la nouvelle loi, mais cette décision n'est pas encore définitive. L'espoir que les autres Municipalités reçoivent également les recettes de pétrole est constatable sur le site de la Confédération Nationale des Municipalités (CNM) qui calcule les «pertes » occasionnées par le maintien de la décision préliminaire du STF. Selon la CNM, « jusqu'à présent, les entités fédérées ont été empêchées de recevoir R\$ 8 milliards, à compter de l'approbation de la Loi n. 12.734/2012 qui 
définit les nouveaux critères de distribution (de façon plus isonomique) ». (www.cnm.prg.br, consulté le 10 octobre 2014).

La CNM est une organisation en faveur des municipalités et dédiée à la lutte pour une « distribution plus isonomique » d'une recette qui s'accroît depuis 15 ans, ce qui suggère l'importance des prélèvements de cette recette pour les budgets municipaux - et également des États-membres. De ce fait, la dispute fédérative en cours suggère que l'intérêt pour l'économie du pétrole est présent au sein de toutes les entités de la fédération, ne se limitant plus aux producteurs.

Dans ces conditions décrites, tout le monde devient plus dépendant de la recette provenant d'une richesse fossile et moins sensibles à la politique énergétique fondée sur les ressources renouvelables. Par ailleurs, la dispute pour les ressources du pétrole change la compréhension de ce qui est considéré comme étant la nature des royalties. Les royalties devraient être une compensation pour les impacts provoqués en fonction de la prospection locale de pétrole. Ces impacts sont environnementaux et sociaux - comme l'insécurité publique et la spéculation foncière. Cette compensation financière par les royalties est nécessaire parce que l'augmentation de l'activité économique, produite par l'exploitation du pétrole, n'est pas suffisante pour les dédommager.

\section{LE NEO-DEVÉLOPPEMENT, LES DROITS SOCIAUX ET LES DROITS DIFFUS}

La raison qui explique le changement des normes de régulation du pétrole est la conviction selon laquelle l'Etat national est capable d'utiliser la richesse produite par l'exploitation du pétrole pour le développement brésilien. Il convient, en ce sens, d'analyser le rôle de l'Etat au Brésil, d'une façon synthétique

Selon Fiori (1995), l’Etat a été un important agent du processus de développement dans l'Amérique Latine et, plus particulièrement au Brésil. Mais il s'agit de «l'Etat devéloppmentiste », non pas de «l'Etat providence » qui caractérise l'Europe de l'après-guerre. Au Brésil, depuis les années 30, avec la crise de l'économie primaire d'exportation, une rupture économique, sociale et politique a eu lieu. Elle a provoqué des transformations qui ont engendré une société à base urbain-industrielle.

Au cours de la période qui va des années 30 aux années 80, l'économie brésilienne s'est amplifiée, ayant devant elle un Etat qui favorisait l'industrialisation et la formation des territoires métropolitaines, sans affecter la structure foncière rurale si concentrée. Les instruments employés ont été les crédits et la fiscalité, mais, également, la création d’un puissant secteur de production publique pour soutenir la structure industrielle du pays. L'objectif de l'Etat était d'agir comme un agent soutenant le niveau de l'activité économique, l'orientant vers les secteurs industriels avec de denses chaines de production, ce qui se reflétait dans l'expansion du marché de travail urbain. 
L'amélioration des conditions de vie était comprise comme la conséquence des avancées économiques, dépendant de la croissance du PIB et fortement liée à la création des opportunités de travail. L'emploi était considéré, par conséquent, comme le droit social par excellence.

Pendant cette période, le «modèle» de développement du Brésil a reçu le nom de " développementiste ", lequel s'opposait à un autre abordage qui défendait un Etat intervenant moins dans la dynamique économique, un libéralisme qui ferait en sorte que les pouvoirs publics s'attachent plus aux objectifs classiques de l'Etat, comme l'établissement de l'ordre et de la justice, se préoccupant de la population la plus vulnérable, une population incapable de trouver des solutions de survie dans le marché.

L'expérience brésilienne suggère que la modernisation sociale et économique a été conduite par l'Etat (Fiori, 1995; Becker \& Egler, 1993; Rego \& Marques, 2003). Après la crise des années 70/80, le développementisme trouverait des limites fiscales pour soutenir la « marche forcée » du progrès de I'économie brésilienne (Castro \& Pires de Souza, 2004). La recrudescence du processus d'inflation, qui atteindrait des indices révélant une hyperinflation, a uniquement été freinée par la succession de plans de stabilisation³. A l'année 1994 arrive à la fin ce modèle du « devéllopementisme ».

Que ce soit en tant qu'Etat du développement, ou en tant qu'État Providence, les années 80 et 90 ont provoqué le recul de l'Etat comme agent économique, sous l'effet de réformes administratives menées dans plusieurs pays (Banque Mondiale, 1997). Ces réformes peuvent être synthétisées par le binôme privatisations et décentralisation : privatisation des entreprises publiques et décentralisation de la manière dont le pouvoir public opère, renforçant les instances régionales et locales de gouvernement (Santos, 2008 ; Restrepo, 2006). Le Brésil n’a pas échappé à ces processus de changement et c'est dans ce contexte que l'entreprise de pétrole brésilienne, la Petrobrás, a perdu son monopole pour l'exploitation de cette richesse minérale. Par la suite, débute un abordage « néolibéral» du développement du pays.

Le néolibéralisme inaugure une nouvelle étape de la dynamique économique mondialisée, marquée par une plus grande indépendance des économies nationales. Dans ce contexte, le rôle des pouvoirs publics commencerait à être évalué selon deux perspectives différentes: la première, critiquant le recul de l'Etat et la seconde expliquant ce recul.

L'abordage critique explore la perte de l'effectivité des droits sociaux qui englobent I'assistance sociale de l'Etat. En d'autres termes, elle dénonce le sous-financement de ses dépenses dans le budget public, comme c'est le cas pour le droit à la santé, à l'éducation, à l'assistance sociale ou au logement. De ce point de vue, l'avancée en matière de droits sociaux observée tout au long du

3 Plano Cruzado (1986), Plano Cruzado II (1987), Plano Verão (1989), Plano Collor (1990) e Plano Real (1994). 
XXe siècle est remise en question et peuvent peut aboutir à une croissante vulnérabilité de la population, qui n'a pas les moyens de sastisfaire ses besoins au niveau des relations économiques du marché.

II s'agit d'un danger aggravé par les effets de la crise de 2008, face à laquelle le pouvoir public a réagi en soutenant les corporations en crise, mais sans se pencher de façon appropriée sur l'augmentation des besoins des travailleurs ayant perdu leurs emplois, et, pour nombre d'entre eux, ayant aussi perdu leurs logements. Cela étant, le recul de l'Etat représente une perte de droits sociaux, y inclus compris le droit au travail, un engagement qui semblait être garanti par les politiques keynésiennes, avec lesquelles la dépression de 1929 a été affrontée.

Cependant il existe des analyses qui visent à expliquer le recul de l'Etat par rapport aux droits sociaux (Rezende, 2013). Dans cette approche, I'attention se portera sur les finances publiques, fortement dépendantes d'une conjoncture de croissance économique, étant donné que les prélèvements fiscaux changeaient en fonction de la performance du PIB. Par conséquent, avec la diminution du rythme de l'expansion économique, la conclusion est qu'il faut ajuster le budget à la capacité de la société de supporter une charge fiscale croissante, au risque de financer les dépenses avec des opérations de crédit, dont le coût a tendance à s'élever si les investisseurs cessent de croire à la croissance de l'économie.

Les dépenses dues aux prestations de l'Etat ont besoin d'être en accord avec la taille du budget public. Si elles sont financées par des opérations de crédit, l'augmentation des dépenses financières (avec le payement d'intérêts du service de la dette publique) dérive de la diminution des dépenses non-financières. De cette façon, une dispute pour le budget de l'Etat s'établit, plaçant les dépenses sociales à l'opposé des autres dépenses publiques (avec l'infrastructure urbaine ou la logistique, par exemple), incluant les dépenses financières avec l'administration de la dette de l'Etat (Almeida, 2013). A partir de la première décennie du XXI siècle, cette dispute s'achèverait par le choix d'un nouveau modèle de développement, appelé le « neo-devéloppement ».

Qu'est-ce donc finalement que le neo-devéloppement ? II s'agit de stimuler la recentralisation de l'organisation de l'Etat, que la structure fédérative ait été conservée. Le renforcement du gouvernement central est considéré comme étant une stratégie permettant de réaliser un projet de développement national, cela s'est produit au cours du développementisme des années 30/70. Un pays comportant un territoire de 8.5 millions de kilomètres carrés présente une grande diversité de caractéristiques sociales, politiques, économiques et de ressources naturelles, ce qui justifierait des politiques de couverture géographique nationale pour que la citoyenneté puisse être expérimentée avec un dénominateur commun minimum entre les Brésiliens. 
En 2003, débute une période marquée par l'ascension du gouvernement du Parti Politique des Travailleurs (PT). Ce parti défend des engagements liés à l'avancée des droits des citoyens et à la participation sociale aux politiques publiques (Ricci, 2013). Après la conquête de la stabilité monétaire, en 1994, des ajustements sont réalisés dans le système financier, qui a commencé à opérer selon un régime post-inflation. L'économie brésilienne a commencé à s'étendre plus intensivement, ce qui rendrait possible l'augmentation des dépenses publiques. De plus, elle a profité du contexte international de forte croissance des commodities, en fonction de la demande chinoise. Dans ces conditions, l'Etat brésilien ajusterait ses politiques en direction d'une dépense sociale plus intensive, modifiant la direction antérieure, caractérisée par l'ajustement fiscal (Monteiro, 2013).

C'est dans ce contexte de prospérité que le pays a été affecté par la crise internationale qui a éclaté suite à la faillite de la Banque Lehman Brothers, en 2008. Par la suite, le gouvernement a plus vivement mis en place des politiques neo-developpementistes, stimulant la consommation pour diminuer les effets de la crise. II entame, de cette façon, une politique d'exonération fiscale des secteurs choisis par le gouvernement, comportant des chaines de production plus denses, capables d'engendrer des opportunités d'emploi, avec l'industrie automobile et de la construction. Cette dernière a profité du lancement du Programme Ma Maison, Ma Vie, responsable de la passation de contrats pour la construction de 3.6 millions de nouveaux logements - la moitié déjà accomplie.

Selon Rudá Ricci (2013), les politiques pendant le gouvernement de Lula (2003/2010) ont été caractérisées par «l'inclusion par la consommation ». La politique de stimulation de la participation sociale, comprise comme un instrument de la conquête de droits, a été abandonnée en faveur de l'augmentation des revenus des travailleurs par l'intermédiaire de l'augmentation du salaire minimum et des transferts conditionnés de ressources. Le résultat a été fructueux par rapport à la manutention de l'activité économique et au recul de la pauvreté d'une grande quantité de gens, environ 30 millions de personnes. Autre manifestation du succès de cette politique a été le niveau élevé d'emplois qui subsistent encore, malgré la perte de l'économie. C'est la diminution de l'expansion économique qui crée la préoccupation selon laquelle ce modèle "neo-développementiste » puisse être en train de s'épuiser, parce qu'il est fondé sur la consommation des familles plutôt que sur l'élévation des indicateurs d'investissement.

Il existe une expectative, à savoir que l'entrée en opération des investissements du pré-sel engendre une richesse qui soutienne ce modèle de développement. Le futur nous dira si ces expectatives se concrétiseront bel et bien, mais il est possible dès à présent de constater l'aggravation de la crise environnementale que la dépendance de l'économie du pétrole provoque. De plus, se pose la question de l'usage croissant d'une source d'énergie fossile dont les richesses déjà créées et 
qui seront encore produites remettent en question la conquête d'une dimension des droits diffus : le droit à l'environnement.

\section{CONCLUSIONS}

Le Brésil est un pays qui présente des indicateurs divergents en question économique et de cadre de vie de sa population : c'est l'une des 10 plus grandes économies mondiales, mais elle occupe la $79^{e}$ position dans le classement qui réunisse 185 pays pour la fixation de l'Indice du Développement Humain (IDH). Cette forte inégalité engendre une certaine différence au niveau de ce que l'on peut attendre de la citoyenneté, par rapport aux pays plus avancés. Dans un pays aux dimensions continentales, cette disparité se manifeste dans les indicateurs socioéconomiques qui opposent les régions au poids économique et démographiques plus ou moins important.

De cette évidence mentionnée ci-dessus découlent des besoins en politiques publiques différents selon les régions. Un État structuré comme une fédération, soutenu par une plus large autonomie régionale/locale, s'intéresse aux régions les plus riches (Sud-est et Sud). Toutefois, les régions présentant de faibles indicateurs socioéconomiques (Nord et Nord-est), intéresse correspondent à/sont susceptibles d'intéresser une structure de gouvernement plus centralisée qui inclue plus de transferts intergouvernementaux pour financer les politiques qui améliorent les conditions locales de vie de la population (Santos, 2008). Le fait de diminuer l'inégalité au niveau des indicateurs sociaux a mené à la croissante centralisation autour le gouvernement fédéral, au détriment de la structure décentralisé de gouvernement institué par la Constitution, en 1988.

Bien qu'il ne soit pas possible d'établir un rapport direct entre ces indicateurs et la sensibilité par rapport aux politiques environnementales, l'urgence d'apporter une réponse aux demandes en droits sociaux affecte l'importance conférée au droit diffus à l'environnement. II me semble que c'est la raison pour laquelle le gouvernement donne la priorité à l'énergie fossile, en l'occurrence le pétrole, au détriment des énergies renouvelables, bien que son importance pour un futur qui n'est plus si lointain soit évidente

Même s'il l'explique, l'abordage du rôle critique de l'Etat demeure. II est vrai que la matrice énergétique brésilienne est liée aux hydroélectriques, ce qui lui confère une bonne position concernant l'usage des énergies renouvelables. Toutefois, la création d'entreprises d'hydroélectricité provoque des problèmes environnementaux entrant en conflit avec la législation qui soutient ce modèle. L'option qui se présente est l'exploitation d'autres sources énergétiques renouvelables. Par rapport à certaines entre elles, le pays compte avec des avantages naturelles, face à l'extension de son territoire (biomasse) et de son climat (énergies solaire et éolique). 
L'Etat brésilien trouve ainsi face à une disjonction entre le besoin évident de mettre en place des politiques qui stimulent l'utilisation des énergies renouvelables et l'émergence de politiques sociales. Financer ces politiques demande des ressources qui ne sont pas alimentées dans une économie qui a diminué le rythme de sa croissance. C'est la raison pour laquelle la dispute fédérative déclenchée par la perspective des recettes de pétrole du pré-sel impose des limites à l'engagement pour le droit diffus à l'environnement, qui profiterait de des énergies renouvelables. Je crois, également qu'il est possible de développer l'usage de ces énergies par l'intermédiaire de politiques incitantes, qui établissent des avantages financiers ou de services prioritaires à ceux qui adhèrent aux énergies renouvelables. Cela semble le chemin pour lequel sont implantées les politiques de stimulation du recours aux énergies renouvelables, comme le suggère la législation récente et les politiques locales, objets d'investigation d'autres chercheurs brésiliens dans le cadre du projet "Energies renouvelables, décentralisation et le rôle des entités fédérées », appuyé par le Programme CAPES/COFECUB.

\section{REFERÊNCIAS BIBLIOGRÁFICAS}

ALMEIDA, Mansueto. Estrutura do gasto público no Brasil: evolução histórica e desafios. In Rezende, F. \& Cunha, A. (org.). A Reforma Esquecida. Orçamento, gestão publica e desenvolvimento. Rio de Janeiro: FGV, 2013.

BANCO MUNDIAL. Relatório sobre o desenvolvimento mundial, 1997. O Estado Num Mundo em Transformação. Oxford University Press para o Banco Mundial, 1997.

BECKER, B. \& Egler, C. Brasil. Uma Nova Potência Regional na Economia-Mundo. Rio de Janeiro: Betrand-Brasil, 1992.

CASTRO, A.B \& Pires de Souza, F.E. A economia brasileira em marcha forçada. São Paulo: Paz e Terra, 2004

DIEGUEZ, Consuelo. Bilhões e Lágrimas. A economia brasileira e seus atores. São Paulo: PortfólioPenguin, 2014.

FIORI, J. L. Em busca do dissenso perdido. Rio de Janeiro: Insight, 1995

MONTEIRO, Jorge Vianna. O ambiente institucional-constitucional da política de gasto público e das escolhas orçamentárias em geral. In Rezende, F. \& Cunha, A. (org.). A Reforma Esquecida. Orçamento, gestão publica e desenvolvimento. Rio de Janeiro: FGV, 2013. 
PIQUET, R. - Os Efeitos Multiplicadores da Indústria Brasileira de Petróleo. In Revista Paranaense de Desenvolvimento. Curitiba, n. 123, p. 81-97, jul/dez/ 2012.

PUGA, F. \& Borça Jr, G. Perspectiva de investimento na indústria 2011-2014. Rio de Janeiro: BNDES, 2011 (Visão do Desenvolvimento, n. 91). Disponível em www.bndes.gov.br/siteBNDES/export/sites/default/fndes_pt/Galerias/Arquivos/conhecimento/visao/ Visao_79.pdf, acesso em 10 de maio de 2011.

REGO, J. M. \& Marques, R. M. (org.) Economia Brasileira. São Paulo: Saraiva, 2003.

RESTREPO, D. (coord). Historias de Descentralización. Transformación del régimen politico y cambio en el modelo de desarrollo. America Latina, Europa y EUA. Bogotá: Universidad Nacional de Colombia. Facultad de Ciencias Económicas, 2006

REZENDE, Fernando. O Estado partido: comportamento das despesas públicas e da dinâmica socioeconômica na Nova República. In Rezende, F. \& Cunha, A. (org.). A Reforma Esquecida. Orçamento, gestão pública e desenvolvimento. Rio de Janeiro: FGV, 2013.

RICCI, Rudá. Lulismo. Da Era dos Movimentos Sociais à ascensão da Nova Classe Média Brasileira. Brasília: Contraponto, 2013.

SANTOS, A.M.S.P. Município, descentralização e território. Rio de Janeiro: Forense, 2008.

SERRA, R. \& Vilani, R. Royalties como ferramenta na construção de uma política de Estado no Rio de Janeiro. In Santos, A. M. S. P., Marafon, G. J. \& Sant'Anna (org.). Rio de Janeiro. Um território em mutação. Rio de Janeiro: Gramma, 2012.

TOSI, A. F. Pré-Sal, Desenvolvimento Industrial e Inovação. In Revista Paranaense de Desenvolvimento. Curitiba, v.34, n. 125 p, jul/dez/ 2013.

Trabalho enviado em 27 de junho de 2015.

Aceito em 29 de junho de 2015. 